\title{
Fratura intertrocantérica do fêmur em um quadril anquilosado: Relato de caso*
}

\section{Intertrochanteric Fracture of the Femur in an Ankylosed Hip: A Case Report}

\author{
Pramod Devkota ${ }^{1}$ Shiraz Ahmad ${ }^{1}$ Ketan C. Pande ${ }^{2}$ \\ ${ }^{1}$ Departmento de Ortopedia e Cirurgia de Traumatismos, Suri Seri \\ Endereço para correspondência Pramod Devkota, MBBS, MS, \\ Begawan Hospital, Kuala Belait, Brunei \\ 2 Departmento de Ortopedia e Cirurgia de Traumatismos, RIPAS \\ Department of Orthopaedics and Trauma Surgery, Suri Seri Begawan \\ Hospital Kuala Belait, Belait District, Brunei Darussalam \\ Hospital, Bandar Seri Begawan, Brunei \\ (e-mail: devkotap@gmail.com).
}

Rev Bras Ortop 2019;54:736-738.

\section{Resumo \\ Palavras-chave \\ - anquilose \\ - fraturas do fêmur \\ - fixação de fratura \\ - articulação do quadril \\ - parafusos ósseos}

\begin{abstract}
A fratura intertrocantérica do fêmur em um quadril anquilosado é extremamente rara. O objetivo do manejo operatório em idosos com fraturas intertrocantéricas é prevenir complicações gerais, manter a mobilidade, e aliviar a dor. O manejo ideal para atingir tal objetivo não é claro. Os autores apresentam o caso de um paciente do sexo masculino de 74 anos com fratura intertrocantérica do fêmur em um quadril anquilosado. A fratura foi tratada cirurgicamente com parafuso de quadril dinâmico e parafuso canulado. Dois anos após a cirurgia, observou-se boa união na fratura, e o paciente deambula de forma independente.
\end{abstract}

Intertrochanteric fractures of the femur in ankylosed hips are extremely rare. The aims of the operative management for elderly patients with intertrochanteric fractures are to prevent general complications, to maintain mobility, and to relieve pain. The optimal management to achieve these goals is not clear. The authors present a case of a 74year-old man with an intertrochanteric fracture of the femur in an ankylosed hip. The fracture was managed surgically with dynamic hip screws and cannulated screws. Two years after the surgery, good union was observed at the fracture, and the patient was ambulating independently.

\section{Introdução}

A incidência de fratura de quadril tem aumentado com o envelhecimento da população, e cerca de $50 \%$ das fraturas de quadril são intertrocantéricas. ${ }^{1}$ Normalmente, o tratamento

\footnotetext{
Trabalho desenvolvido no Departmento de Ortopedia e Cirurgia de Traumatismos, Suri Seri Begawan Hospital, Kuala Belait, Brunei. Originalmente Publicado por Elsevier Editora Ltda.
}

recebido

01 de Outubro de 2017

aceito

23 de Novembro de 2017
DOI https://doi.org/

10.1016/j.rboe.2017.11.016. ISSN $0102-3616$. cirúrgico é ideal em pacientes idosos com fraturas intertrocantéricas, e o objetivo é evitar complicações gerais, manter a mobilidade, e aliviar a dor. ${ }^{2}$ Há poucos relatos na literatura sobre a fratura intertrocantérica de fêmur em um quadril com anquilose; ${ }^{2,3}$ portanto, a literatura sobre essas fraturas em quadris anquilosados é escassa.

Relatamos um caso de fratura intertrocantérica de fêmur em um quadril com anquilose tratada com parafusos dinâmicos (PDs).
Copyright $(2019$ by Sociedade Brasileira License terms de Ortopedia e Traumatologia. Published by Thieme Revinter Publicações Ltda, Rio de Janeiro, Brazil 


\section{Relato de Caso}

Um homem de 74 anos de idade foi atendido no prontosocorro com dor no quadril direito um dia após uma queda. Ao exame, havia sensibilidade na região trocantérica superior direita e incapacidade de elevação ativa do membro inferior direito. Havia um discreto aumento de volume na região intertrocantérica direita com alguma equimose. 0 paciente não se queixava de dor com irradiação ou dormência, e não era capaz de andar e sustentar peso devido à dor.

O paciente havia sido submetido a uma cirurgia no quadril direito 20 antes, e, desde então, caminhava com andador e apresentava claudicação. $O$ paciente estava em tratamento para hipertensão e hiperlipidemia. Além disso, havia sido tratado por câncer de cólon alguns anos antes.

Os músculos da região do quadril estavam atrofiados. A radiografia pélvica mostrou necrose avascular com suspeita de infecção na cabeça do fêmur e anquilose do quadril. Uma pequena placa foi observada in situ na região acetabular, com

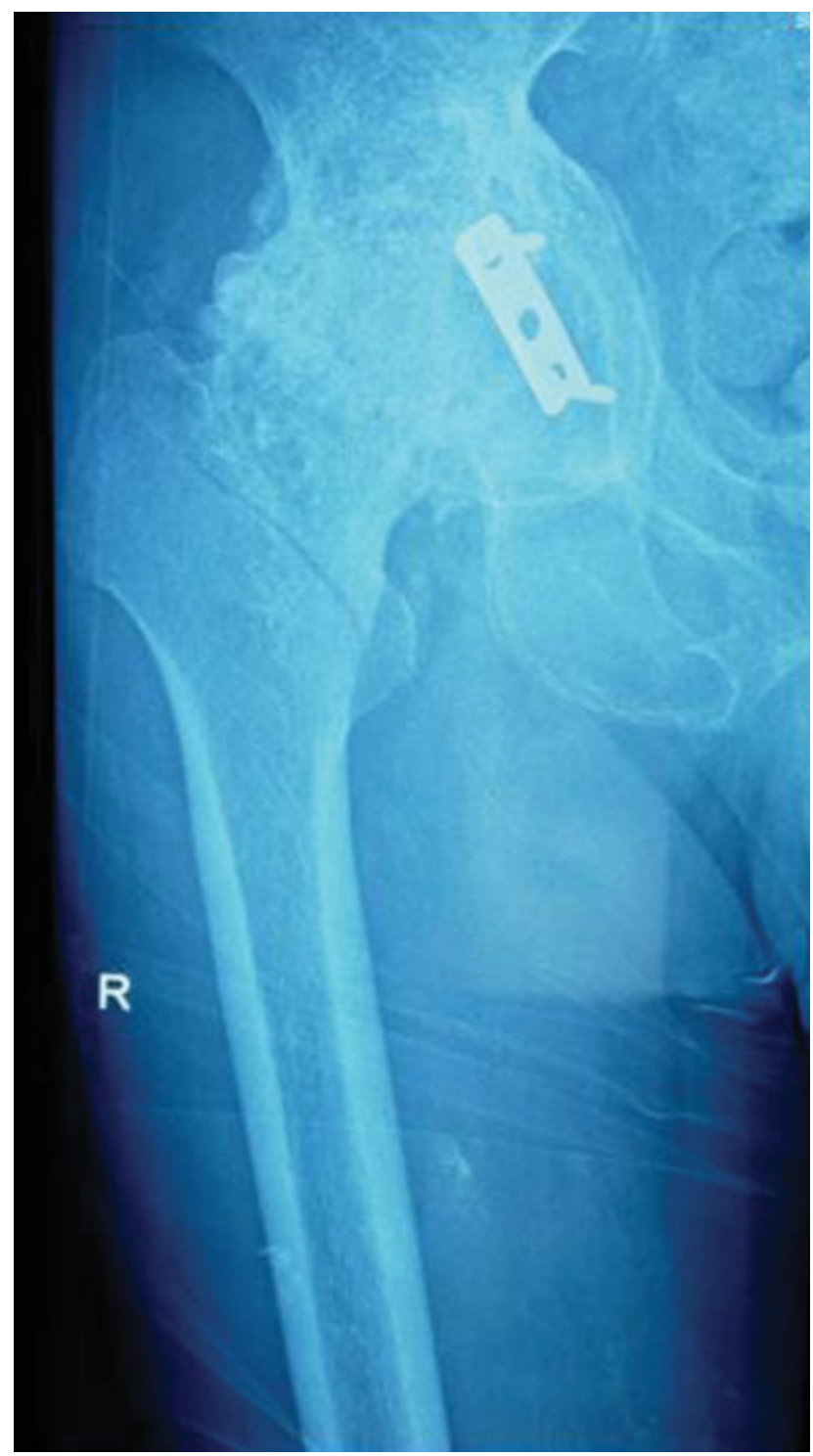

Fig. 1 Fratura intertrocantérica não deslocada do fêmur em um quadril com anquilose. fratura não deslocada da região intertrocantérica do fêmur (-Fig. 1).

A fratura foi tratada com PDs e um parafuso canulado para fixação do acetábulo. $O$ paciente recebeu alta no $21^{\circ}$ dia de pós-operatório. A não sustentação de peso foi aconselhada por seis semanas após o procedimento. A sustentação parcial de peso foi permitida após a sétima semana, e o paciente voltou a caminhar com sustentação total após três meses de cirurgia. O paciente continuou em acompanhamento regular, e, 18 meses após a cirurgia, a fratura estava consolidada, e ele estava satisfeito com o resultado (-Fig. 2).

\section{Discussão}

A fratura intertrocantérica do fêmur no quadril anquilosado é extremamente rara, com poucos casos relatados na literatura. O tratamento não operatório está associado a muitas complicações relacionadas à imobilização prolongada, e a

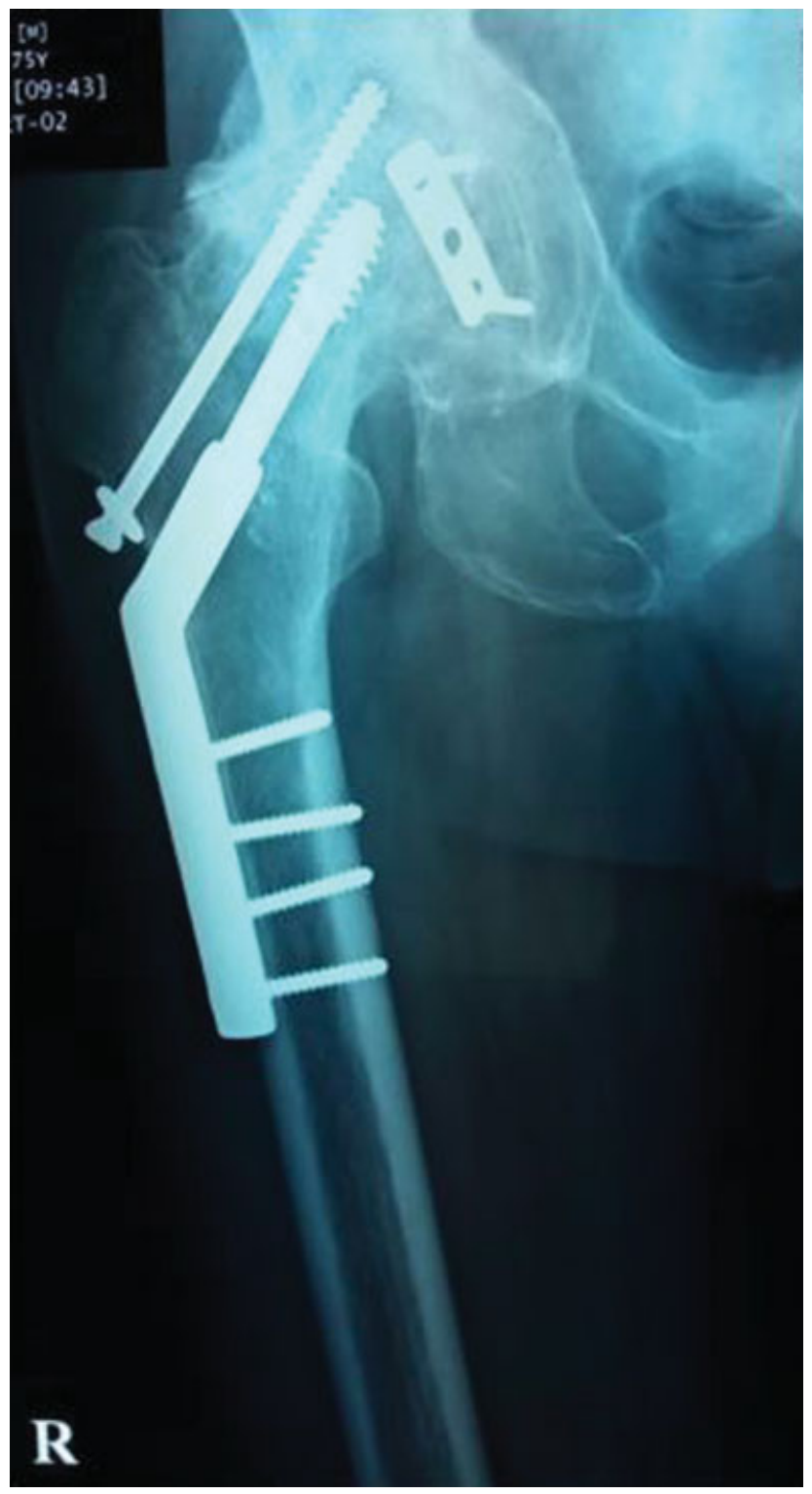

Fig. 2 Um ano e meio após a cirurgia: fratura consolidada e implantes in situ. 
opção cirúrgica é melhor se o paciente estiver fisiologicamente bem.

A fratura era instável devido à fusão da articulação do quadril na região proximal ao local da fratura e à presença do longo braço de alavanca distal do membro inferior. ${ }^{2} \mathrm{O}$ caso não é fácil, e há várias opções para a fixação. Utilizamos PDs e um parafuso canulado para perfuração acetabular e obtenção da estabilidade adequada. Acreditamos que os PDs não eram suficientes devido ao braço da alavanca distal do membro inferior e à fusão do quadril, que pressionam o local da fratura, e podem provocar o insucesso do implante.

A artroplastia total do quadril (ATQ) é comumente usada em casos com fusão da articulação do quadril, mas é tecnicamente difícil devido à ausência de referências cirúrgicas. ${ }^{4}$ Entretanto, neste caso, considerando as más condições musculares em volta do quadril e da coxa e o histórico recente de tratamento de câncer de cólon, escolhemos os PDs. Existem casos relatados de aplicação apenas de haste intramedular ${ }^{2,3}$ e parafusos canulados, com bons resultados. ${ }^{5}$ Neste caso, a opção de haste intramedular não foi considerada devido à má condição muscular em volta do quadril e à natureza não deslocada da fratura intertrocantérica. Embora o caminhar com sustentação do peso tenha sido iniciado posteriormente aos casos intramedulares supramencionados, não houve intercorrências durante a cicatrização da fratura e o restante da reabilitação desse paciente. Acreditamos que o PD do quadril também é uma opção para a fratura intertrocantérica em indivíduos com anquilose de quadril.

Conflitos de Interesse

Os autores declaram não haver conflitos de interesse.

\section{Referências}

1 Bhandari M, Schemitsch E, Jönsson A, Zlowodzki M, Haidukewych GJ. Gamma nails revisited: gamma nails versus compression hip screws in the management of intertrochanteric fractures of the hip: a meta-analysis. J Orthop Trauma 2009;23(06):460-464

2 Ishimaru D, Nozawa S, Maeda M, Shimizu K. Intertrochanteric fracture of the ankylosed hip joint treated by a gamma nail: a case report. Case Rep Orthop 2012;2012:278156

3 Manzotti A, Confalonieri N, Pullen C. Intertrochanteric fracture of an arthrodesed hip. JBone Joint Surg Br 2007;89(03):390-392

4 Kilgus DJ, Amstutz HC, Wolgin MA, Dorey FJ. Joint replacement for ankylosed hips. J Bone Joint Surg Am 1990;72(01):45-54

5 Font-Vizcarra L, Carreño AM, Prat S, Muñoz-Mahamud E, Camacho P, Casanova L. Less invasive fixation of an interthrocanteric fracture in an ankylosed hip with cannulated screws: a case report. Hip Int 2010;20(04):565-567 